\title{
The Zeros of Orthogonal Polynomials and Markov-Bernstein Inequalities for Jacobi-Exponential Weights on $(-1,1)$
}

\author{
Rong Liu \\ College of Computer and Information Sciences, Fujian Agriculture and Forestry University, Fuzhou, Fujian 350002, China \\ Correspondence should be addressed to Rong Liu; chensi1983@163.com
}

Received 18 January 2020; Revised 26 June 2020; Accepted 30 July 2020; Published 24 August 2020

Academic Editor: Markos Koutras

Copyright (c) 2020 Rong Liu. This is an open access article distributed under the Creative Commons Attribution License, which permits unrestricted use, distribution, and reproduction in any medium, provided the original work is properly cited.

Let $U(x)=\prod_{i=1}^{r}\left|x-t_{i}\right|^{p_{i}}, 0<p<\infty,-1=t_{r}<t_{r-1}<\cdots<t_{2}<t_{1}=1, r \geq 2, p_{i}>-1 / p, i=1,2, \ldots, r$, and $W=e^{-Q(x)}$ where $Q:(-1,1) \longrightarrow[0, \infty)$. We give the estimates of the zeros of orthogonal polynomials for the Jacobi-Exponential weight $W U$ on $(-1,1)$. In addition, Markov-Bernstein inequalities for the weight $W U$ are also obtained.

\section{Introduction and Results}

Let $w$ be a weight in $\mathbf{I}:=(a, b),-\infty \leq a<0<b \leq \infty$, for which the moment problem possesses an unique solution. $\mathbf{P}_{n}$ stands for the set of polynomials of degree at most $n \cdot\|\cdot\|_{L_{p}(\mathrm{I})}$ is an usual (weighted) $L_{p}$ (quasi) norm on interval $\mathbf{I}$.

Assume that $W=e^{-Q}$ where $Q: \mathbf{I} \longrightarrow[0, \infty)$ is continuous. $W$ is an exponential weight on I. Also, let $0<p<$ $\infty, a \leq t_{r}<t_{r-1}<\cdots<t_{2}<t_{1} \leq b, p_{i}>-1 / p, i=1,2, \ldots, r$, and

$$
U(x)=\prod_{i=1}^{r}\left|x-t_{i}\right|^{p_{i}},
$$

where $U$ is a generalized Jacobi weight on $\mathbf{I}$. The combination $W U$ is called a Jacobi-exponential weight on $\mathbf{I}$. This paper deals with the zeros of orthogonal polynomials and Markov-Bernstein inequalities for Jacobi-exponential weights.

The letters $c, C_{0}, C_{1}, \ldots$ stand for positive constants independent of variables and indices, unless otherwise indicated and their values may be different at different occurrences, even in subsequent formulas. Moreover, $C_{n} \sim D_{n}$ means that there are two constants $c_{1}$ and $c_{2}$ such that $c_{1} \leq C_{n} / D_{n} \leq c_{2}$ for the relevant range of $n$. We write $c=c(\lambda)$ or $c \neq c(\lambda)$ to indicate dependence on or independence of a parameter $\lambda$.
Definition 1 (see [1], Definition 1.7, p. 14). Given $c, t \geq 0$ and a non-negative Borel measure $v$ with compact support in $\mathbf{C}$ and total mass $\leq t$, we say that

$$
P(z):=c \exp \left(\int \ln |z-s| \mathrm{d} v(s)\right),
$$

is an exponential of a potential of mass $\leq t$. We denote the set of all such $P$ by $\mathscr{P}_{t}$.

We note that for $P \in \mathbf{P}_{n},|P| \in \mathscr{P}_{t}, t \geq n$.

Definition 2 (see [1], p. 19). Let $w$ be a weight in I. For $0<p<\infty$, generalized Christoffel functions with respect to $w$ for $z \in \mathbf{C}$ are defined by

$$
\lambda_{p, n}(w ; z)=\inf _{P \in P_{n}}\left(\frac{\|P w\|_{L_{p}(I)}}{|P(z)|}\right)^{p} .
$$

For $p=\infty$, generalized Christoffel functions with respect to $w$ for $z \in \mathbf{C}$ are defined by

$$
\lambda_{\infty, n}(w ; z)=\inf _{P \in P_{n}} \frac{\|P w\|_{L_{\infty}(I)}}{|P(z)|} .
$$

Moreover, for the classical Christoffel function $\lambda_{n}\left(w^{2} ; x\right)$ with respect to $w^{2}$, we have

$$
\lambda_{n}\left(w^{2} ; x\right)=\inf _{P \in P_{n-1}} \int_{I} \frac{(P w)^{2}(t) \mathrm{d} t}{P^{2}(x)}=\lambda_{2, n-1}(w ; x) .
$$


A function $f:(c, d) \longrightarrow(0, \infty)$ is said to be quasi-increasing (or quasidecreasing) if there exists $C>0$ such that $f(x) \leq($ or $\geq) C f(y), c<x \leq y<d$.

Definition 3. (see [1], pp. 10-12). Let $a<0<b$. Assume that $W=e^{-Q}$ where $Q: I \longrightarrow[0, \infty)$ satisfies the following properties:

(a) $Q^{\prime} \in C($ I $)$ and $Q(0)=0$.

(b) $Q^{\prime}$ is nondecreasing in $\mathbf{I}$.

(c) $\lim _{x \longrightarrow a_{+}^{+}} Q(x)=\lim _{x \longrightarrow b_{-}} Q(x)=\infty$.

(d) The function

$$
T(x):=\frac{x Q^{\prime}(x)}{Q(x)}, \quad x \neq 0
$$

is quasidecreasing in $(a, 0)$ and quasi-increasing in $(0, b)$, respectively. Moreover, $T(x) \geq \Lambda>1, x \in$ $I \backslash\{0\}$.

(e) There exists $\epsilon_{0} \in(0,1)$ such that for $y \in \mathbf{I} \backslash\{0\}$,

$$
T(y) \sim T\left(y\left[1-\frac{\epsilon_{0}}{T(y)}\right]\right) \text {. }
$$

Then, we write $W \in \mathscr{F}$. (f) Furthermore, assume that there exist $C, \epsilon_{1}>0$ such that for all $x \in \mathbf{I} \backslash\{0\}$,

$$
\int_{x-\epsilon_{1}|x| / T(x)}^{x} \frac{\left|Q^{\prime}(s)-Q^{\prime}(x)\right|}{|s-x|^{3 / 2}} \mathrm{~d} s \leq C\left|Q^{\prime}(x)\right|\left[\frac{T(x)}{|x|}\right]^{1 / 2} .
$$

Then, we write $W \in \mathscr{F}(\operatorname{Lip}(1 / 2))$.

In addition, let $W \in \mathscr{F}$. Assume that there exist $C, \epsilon_{1}>0$ such that for all $x \in \mathbf{I} \backslash\{0\}$,

$$
\int_{x-\epsilon_{1}|x| / T(x)}^{x+\epsilon_{1}|x| / T(x)} \frac{Q^{\prime}(s)-Q^{\prime}(x)}{s-x} \mathrm{~d} s \leq C\left|Q^{\prime}(x)\right| .
$$

Then, we write $W \in \mathscr{F}$ (Dini).

For $W \in \mathscr{F}$ and $t>0$, the Mhaskar-Rahmanov-Saff numbers $a_{-t}:=a_{-t}(Q)<0<a_{t}:=a_{t}(Q)$ are defined by the equations

$$
\begin{aligned}
& t=\frac{1}{\pi} \int_{a_{-t}}^{a_{t}} \frac{x Q^{\prime}(x)}{\left[\left(x-a_{-t}\right)\left(a_{t}-x\right)\right]^{1 / 2}} \mathrm{~d} x, \\
& 0=\frac{1}{\pi} \int_{a_{-t}}^{a_{t}} \frac{\mathrm{Q}^{\prime}(x)}{\left[\left(x-a_{-t}\right)\left(a_{t}-x\right)\right]^{1 / 2}} \mathrm{~d} x .
\end{aligned}
$$

Put for $t>0$,

$$
\begin{aligned}
& \Delta_{t}:=\Delta_{t}(Q):=\left[a_{-t}, a_{t}\right], \\
& \delta_{t}:=\delta_{t}(Q):=\frac{1}{2}\left(a_{t}+\left|a_{-t}\right|\right), \\
& \eta_{ \pm t}:=\eta_{ \pm t}(Q):=\left[t T\left(a_{ \pm t}\right) \sqrt{\left.\frac{\left|a_{ \pm t}\right|}{\delta_{t}}\right]^{-2 / 3},}\right. \\
& \varphi_{t}(x):=\varphi_{t}(Q ; x):= \begin{cases}t \sqrt{\left[\left|x-a_{-t}\right|+\left|a_{-t}\right| \eta_{-t}\right]\left[\left|x-a_{t}\right|+a_{t} \eta_{t}\right]}, & x \in\left[a_{-t}, a_{t}\right], \\
\varphi_{t}\left(a_{t}\right), & x \in\left(a_{t}, b\right), \\
\varphi_{t}\left(a_{-t}\right), & x \in\left(a, a_{-t}\right), \\
\mathbf{J}_{L, t}:=J_{L, t}(Q):=\left[a_{-t}\left(1+L \eta_{-t}\right), a_{t}\left(1+L \eta_{t}\right)\right], & L>0, \\
\mathbf{K}_{L, t}:=K_{L, t}(Q):=\left[-1+L\left(1+a_{-t}\right), 1-L\left(1-a_{t}\right)\right], & L>1 .\end{cases}
\end{aligned}
$$

In 1994 and 2001, Levin and Lubinsky [1, 2] discussed orthogonal polynomials for exponential weights $W^{2}$ on $[-1,1]$ and $(a, b), a<0<b$, respectively. Then, they $[3,4]$ dealt with exponential weights $x^{2 \alpha} W(x)^{2}, \alpha>-1 / 2$, in $[0, b)$. Kasuga and Sakai [5] considered generalized Freud weights $|x|^{2 \alpha} W(x)^{2}$ in $(-\infty, \infty)$. Recently, we discussed generalized Jacobi-exponential weights $U W[6,7]$, which centered on the distribution of zeros and the estimates of the generalized Christoffel functions, respectively. Shi [8] also considered
Jacobi-exponential weights $U W$ and subsequently dealt with a particular case $\left(1-x^{2}\right)^{\rho} e^{-Q(x)}$ on $(-1,1)$ in [9].

For the weight $U W$ on $(-1,1)$, its $n^{\text {th }}$ orthogonal polynomial $p_{n}\left((U W)^{2}, x\right)$ has zeros $\left\{x_{k n}\right\}_{k=1}^{n}$, where $-1<x_{n n}<x_{n-1, n}<\cdots<x_{2 n}<x_{1 n}<1$.

The estimates of the zeros [6] are based on the condition $a<t_{r}<t_{r-1}<\cdots<t_{2}<t_{1}<b$. In [6], we did not consider the case when $a=t_{r}$ and $t_{1}=b$, which is different from $a<t_{r}, t_{1}<b$. In this paper, we discuss orthogonal 
polynomials for generalized Jacobi-exponential weights in the case $-1=t_{r}<t_{r-1}<\cdots<t_{2}<t_{1}=1$.

Mastroianni and Totik in [10] gave the estimates of the spacing of zeros for doubling weights; in general, however, Jacobi-exponential weights $U W$ are not doubling weights, so our main result (Theorem 4) cannot follow from it. The distribution of the zeros of orthogonal polynomials plays an important role in weighted approximation, for example, Mastroianni and Notarangelo [11, 12] applied the zeros for exponential weight on $(-1,1)$ and the real semiaxis to deal with Lagrange interpolation processes on corresponding interval, respectively.

We construct the following weight:

$$
\begin{aligned}
Q^{*}(x) & :=Q(x)+p_{0} q(x), \\
q(x) & :=-\ln \left(1-x^{2}\right), W^{*}(x):=e^{-Q^{*}(x)}, \\
p_{0} & :=\min \left\{p_{1}, p_{r}, 0\right\}, \\
U^{*}(x) & :=\left(1-x^{2}\right)^{-p_{0}} U(x) .
\end{aligned}
$$

Some corresponding notations for $W^{*}(x)$ are also needed:

$$
\begin{aligned}
a_{ \pm t}^{*} & :=a_{ \pm t}\left(Q^{*}\right), \\
\eta_{ \pm t}^{*} & :=\eta_{ \pm t}\left(Q^{*}\right), \\
\Delta_{t}^{*} & :=\Delta_{t}\left(Q^{*}\right) \\
\delta_{t}^{*} & :=\delta_{t}\left(Q^{*}\right), \\
\varphi_{t}^{*}(x) & :=\varphi_{t}\left(Q^{*} ; x\right), \\
\rho^{*} & :=\rho\left(U^{*}\right):=p_{1}+p_{r}-2 p_{0}+\sum_{i=2}^{r-1} \max \left\{p_{i}, 0\right\}, \\
\mathbf{J}_{L, t}^{*} & :=\mathbf{J}_{L, t}\left(Q^{*}\right), \\
\bar{U}_{t}(x) & :=\prod_{i=1}^{r}\left(\left|x-t_{i}\right|+\frac{1}{t}\right)^{p_{i}}, \\
U_{t}^{*}(x) & =\left[\left(1-x^{2}\right)^{1 / 2}+\frac{1}{t}\right]^{-2 p_{0}} \bar{U}_{t}(x) .
\end{aligned}
$$

In all that follows, I denotes the open interval $(-1,1)$.

Theorem 1 (see [7], Theorem 1.7). Let $W \in \mathscr{F}(\operatorname{Lip}(1 / 2))$ and $0<p<\infty$. Assume that

$$
x \stackrel{\lim }{\longrightarrow} 0 \frac{x}{Q^{\prime}(x)}=\lambda<\frac{\Lambda-1}{2 \Lambda\left|p_{0}\right|}, \quad p_{0} \neq 0,
$$

and for some constant $\mu$ satisfying

$$
2 \lambda\left|p_{0}\right|<\mu<1-\frac{1}{\Lambda}
$$

the function $\bar{Q}(x)=\mu Q^{\prime}(x)+p_{0} q^{\prime}(x)$ is nondecreasing in $\mathbf{I}$. (a) Then there exists $n_{0}>0$ such that for $n \geq n_{0}$ and $x \in \mathbf{J}_{L, n}^{*}$ with $L>0$, the relation

$$
\left.\lambda_{p, n}(U W ; x)\right) \sim \varphi_{n}^{*}(x) \bar{U}_{n}(x)^{p} W(x)^{p} .
$$

uniformly holds.

(b) Furthermore, there exists $n_{1}>0$ such that for $n \geq n_{1}$ and $x \in \mathrm{I}$, the relation

$$
\lambda_{p, n}(U W ; x) \geq C \varphi_{n}^{*}(x) U_{n}^{*}(x)^{p} W^{*}(x)^{p},
$$

uniformly holds.

By specializing to $p=2$ of Theorem 1 , we obtain estimates for the classical Christoffel functions.

Corollary 1. Assume that the conditions of Theorem 1 hold.

(a) Then, there exists $n_{0}>0$ such that for $n \geq n_{0}$ and $x \in \mathbf{J}_{L, n}^{*}$ with $L>0$, the relation

$$
\begin{aligned}
\lambda_{n}\left((U W)^{2} ; x\right) & \sim \varphi_{n}^{*}(x) \bar{U}_{n}(x)^{2} W(x)^{2} \\
& \sim \varphi_{n}^{*}(x) U_{n}^{*}(x)^{2} W^{*}(x)^{2},
\end{aligned}
$$

uniformly holds.

(b) Furthermore, if $p_{0} \leq 0$, there exists $C, n_{1}>0$ such that for $n \geq n_{1}$ and $x \in \mathbf{I}$, the relation

$$
\lambda_{n}\left((U W)^{2} ; x\right) \geq C \varphi_{n}^{*}(x) \bar{U}_{n}(x)^{2} W(x)^{2},
$$

uniformly holds.

Our results will mainly center on the zeros of orthogonal polynomials for Jacobi-exponential weights $U W$ and Markov-Bernstein inequalities.

Theorem 2. Let $W=e^{-Q(x)}$, where $Q: \mathbf{I} \longrightarrow[0, \infty)$ is convex with $Q(a+)=Q(b-)=\infty$ and $Q(x)>Q(0)=$ $0, x \in I \backslash\{0\} . \quad$ Let $0<p<\infty, \quad P \in \mathscr{P}_{t-\rho^{*}-2 / p} \backslash\{0\}, \quad p_{i} \geq 0$, $i=2, \ldots, r-1$. Assume that relation (16) is valid and $\bar{Q}$ is nondecreasing in $\mathbf{I}$. Then,

$$
\|P U W\|_{L_{p}\left(I \backslash \Delta_{t}^{*}\right)}<\|P U W\|_{L_{p}\left(\Delta_{t}^{*}\right)} .
$$

In particular, this holds for not identically vanishing polynomials $P$ of degree $\leq t-\rho^{*}-(2 / p)$. For $p=\infty$, (22) holds with $<$ replaced by $\leq$.

Theorem 3. Let $0<p \leq \infty$ and $p_{i} \geq 0, i=2, \ldots, r-1$. Assume that relation (16) is valid and $\bar{Q}$ is nondecreasing in $\mathbf{I}$.

(a) Let $W \in \mathscr{F}(\operatorname{Lip}(1 / 2))$. Then, for $t \geq 1$ and $P \in \mathscr{P}_{t-\rho^{*}}$,

$$
\left\|(P U W)^{\prime} \varphi_{t}^{*}\right\|_{L_{p}(\mathbf{I})} \leq C\|P U W\|_{L_{p}(\mathbf{I})} \text {. }
$$

(b) Let $0<\alpha<1$ and $W \in \mathscr{F}$ (Dini). Then, for $t \geq 1$ and $P \in \mathscr{P}_{t-\rho^{*}}$,

$$
\left\|(P U W)^{\prime} \varphi_{t}^{*}\right\|_{L_{p}\left(\Delta_{\alpha t}^{*}\right)} \leq C\|P U W\|_{L_{p}(\mathbf{I})} .
$$


Theorem 4. Let $W \in \mathscr{F}(\operatorname{Lip} 1 / 2)$ and $p_{i}>(-1 / 2), 1 \leq i \leq r$. Assume that relation (16) is valid, $\bar{Q}$ is nondecreasing in $\mathbf{I}$, and

$$
\varphi_{t}^{*}(x)=O(1), \quad t \longrightarrow \infty .
$$

(a) Then, for large enough $n$ and $1 \leq k \leq n-1$,

$$
x_{k n}-x_{k+1, n} \leq c \varphi_{n}^{*}\left(x_{k n}\right) \text {. }
$$

(b) Furthermore, if $r=2$, then for large enough $n$ and $1 \leq k \leq n-1$

$$
x_{k n}-x_{k+1, n} \sim \varphi_{n}^{*}\left(x_{k n}\right) .
$$

Remark 1. By [7], (Lemma 2.12), for zeros $x_{k n}, x_{k+1, n} \in \mathbf{K}_{L, n}$ with $L>1$, the statement (a) of Theorem 4 is valid and $\leq$ can be replaced by $\sim$.

Theorem 5. Assume that the assumptions of Theorem 2 hold. Then,

$$
a_{-n-\rho^{*}-1 / 2}^{*}<x_{n n}<x_{n-1, n}<\cdots<x_{2 n}<x_{1 n}<a_{n+\rho^{*}+1 / 2}^{*} .
$$

Theorem 6. Let $W \in \mathscr{F}(\operatorname{Lip}(1 / 2))$. Assume that relation (16) is valid and $\bar{Q}$ is nondecreasing in $\mathbf{I}$.

(a) Then,

$$
\begin{aligned}
& x_{1 n} \geq a_{n}^{*}\left(1-c \eta_{n}^{*}\right), \\
& x_{n n} \leq a_{-n}^{*}\left(1-c \eta_{-n}^{*}\right) .
\end{aligned}
$$

(b) Furthermore, if $p_{i} \geq 0, i=2, \ldots, r-1$, then for large enough $n$,

$$
\begin{aligned}
& 1-\frac{x_{1 n}}{a_{n}^{*}} \sim \eta_{n}^{*}, \\
& 1-\frac{x_{n n}}{a_{-n}^{*}} \sim \eta_{-n}^{*} .
\end{aligned}
$$

We prove Theorems 2-4 and Theorem 6 in Section 3, but first we need some auxiliary lemmas and the proofs of Corollary 1 and Theorem 5, which are presented in Section 2.

\section{Auxiliary Lemmas}

Lemma 1 (see [1], Theorem 4.1, p. 95). Let $W=e^{-Q(x)}$, where $Q: \mathbf{I} \longrightarrow[0, \infty)$ is convex with $Q(a+)=Q(b-)=\infty$ and $Q(x)>Q(0)=0, x \in I \backslash\{0\}$. Let $0<p \leq \infty$ and $P \in \mathscr{P}_{t-2 / p} \backslash\{0\}$. Then,

$$
\|P W\|_{L_{p}\left(\mathrm{I} \backslash \Delta_{t}\right)}<\|P W\|_{L_{p}\left(\Delta_{t}\right)}
$$

In particular, this holds for not identically vanishing polynomials $P$ of degree $\leq t-2 / p$. For $p=\infty$, (31) holds with $<$ replaced by $\leq$.

Lemma 2 (see [1], Theorem 10.1, p. 293). Let $0<p \leq \infty$.

(a) Let $W \in \mathscr{F}(\operatorname{Lip}(1 / 2))$. Then, for $t \geq 1$ and $P \in \mathscr{P}_{t}$,

$$
\left\|(P W)^{\prime} \varphi_{t}\right\|_{L_{p}(\mathbf{I})} \leq C\|P W\|_{L_{p}(\mathbf{I})}
$$

(b) Let $W \in \mathscr{F}$ (Dini) and $0<\alpha<1$. Then, for $t \geq 1$ and $P \in \mathscr{P}_{t}$,

$$
\left\|(P W)^{\prime} \varphi_{t}\right\|_{L_{p}\left(\Delta_{\alpha t}\right)} \leq C\|P W\|_{L_{p}(\mathbf{I})} .
$$

Lemma 3 (see [7], Lemma 2.13). Let $\mathbf{I}=(-1,1)$ and $W \in \mathscr{F}($ Lip $(1 / 2))$. Assume that (16) is valid and $\bar{Q}$ is nondecreasing in I. Then, $W^{*} \in \mathscr{F}(\operatorname{Lip}(1 / 2))$.

Lemma 4. For fixed index $k, 1 \leq k \leq n-1$, let $\mathbf{I}_{k}=\left[x_{k+1, n}, x_{k n}\right]$. Let $j, 1 \leq j \leq r$, satisfy

$$
\min _{x \in \mathbf{I}_{k}}\left|x-t_{j}\right|=\min _{1 \leq i \leq r} \min _{x \in \mathbf{I}_{k}}\left|x-t_{i}\right| \text {. }
$$

Then,

$$
\begin{aligned}
\prod_{i \neq j}\left|x_{\kappa n}-t_{i}\right|^{p_{i}} \sim \prod_{i \neq j}\left(\left|x_{\kappa n}-t_{i}\right|+\frac{1}{n}\right)^{p_{i}} \\
\sim \prod_{i \neq j}\left|x-t_{i}\right|^{p_{i}}, \quad x \in \mathbf{I}_{k}, \kappa=k, k+1 .
\end{aligned}
$$

Proof. Following the argument in the proof of Lemma 2.5 in [6], we get (35) by replacing $\delta_{n} / n$ with $1 / n$.

Lemma 5. Let $W \in \mathscr{F}$ and (25) be valid. Then, there exists $t_{0}>0$ such that for $t>t_{0}$ and for each index $j, 2 \leq j \leq r-1$,

$$
\left|x-t_{j}\right|+\frac{1}{t} \sim\left|x-t_{j}\right|+\frac{\delta_{t}}{t} \sim\left|x-t_{j}\right|+\varphi_{t}(x),
$$

holds uniformly for $x \in \mathbf{I}$.

Proof. By (1.55) in [1], we see that there exists $t_{0}>0$ such that for $t>t_{0},\left|a_{ \pm t}\right| \sim 1$, so we have $\delta_{t} \sim 1$ for $t>t_{0}$. Also, notice that $-1<t_{r-1}<\cdots<t_{2}<1$, and (36) follows from Lemma 2.7 in [6].

Lemma 6. Let $W \in \mathscr{F}(\operatorname{Lip}(1 / 2))$. Assume that relation (16) is valid and $\bar{Q}$ is nondecreasing in $\mathbf{I}$. Then, there exists $L>0$ such that for $t$ large enough,

$$
a_{t+\rho^{*}+(1 / 2)}^{*} \leq a_{t}^{*}\left(1+L \eta_{t}^{*}\right) \text {. }
$$

Proof. By Lemma 3.11(a) in [1], for $t>0$,

$$
\frac{a_{t+\rho^{*}+(1 / 2)}^{*}}{a_{t}^{*}}-1 \sim \frac{1}{T\left(a_{t}^{*}\right)} \cdot \frac{\rho^{*}+(1 / 2)}{t} .
$$

Fix $t_{0}=\left(\rho^{*}+1 / 2\right)^{3}$; for $t \geq t_{0}$, we have

$$
\frac{1}{T\left(a_{t}^{*}\right)} \cdot \frac{\rho^{*}+(1 / 2)}{t} \leq t^{-2 / 3} T^{-1}\left(a_{t}^{*}\right) \text {. }
$$

On the other hand, using Definition 2 of $\eta_{t}^{*}$, we obtain 


$$
\eta_{t}^{*} \geq C T^{-1}\left(a_{t}^{*}\right) t^{-2 / 3}
$$

as $a_{t}^{*} \sim \delta_{t}^{*}$ and $T\left(a_{t}^{*}\right)>1$.

Thus, by (38), for large enough $t$,

$$
\frac{a_{t+\rho^{*}+(1 / 2)}^{*}}{a_{t}^{*}}-1 \leq L \eta_{t}^{*} .
$$

This yields (37).

Since the last lemma is based on the results of Corollary 1 and Theorem 5, we present the proofs of Corollary 1 and Theorem 5 first.

Proof of Corollary 1. It is the special case of Theorem 1 when $p=2$ we use (5) and the relation $\varphi_{n}^{*} \sim \varphi_{n-1}^{*}$ in I from Lemma 9.7 [1]. We also see that $\bar{U}_{n} \sim \bar{U}_{n-1}$ for $n$ large enough.

Proof of Theorem 5. By Lemma 3, $W^{*}$ satisfies the conditions of $W$. For $U^{*}=|x+1|^{p_{r}-p_{0}}|x-1|^{p_{1}-p_{0}} \prod_{i=2}^{r-1}\left|x-t_{i}\right|^{p_{i}}$, we have $p_{1}-p_{0} \geq 0, p_{r}-p_{0} \geq 0$. Meanwhile, $p_{i} \geq 0, i=2, \ldots$, $r-1$, so (28) follows directly from Theorem 1.9 in [6].

Lemma 7. Let $W \in \mathscr{F}(\operatorname{Lip}(1 / 2))$ and $r=2$. Assume that relation (17) is valid and $\bar{Q}$ is nondecreasing in $\mathbf{I}$. Let $\ell_{j n} \in \mathbf{P}_{n-1}$ be the fundamental polynomials of Lagrange Interpolation at the zeros $p_{n}\left((U W)^{2}, x\right)$ satisfying $\ell_{j n}\left(x_{k n}\right)=\delta_{k j}$. Then, for each index $j, 1 \leq j \leq n$ and large enough $n$,

$$
\begin{aligned}
& \left|\ell_{j n} W U\right|(x)(W U)^{-1}\left(x_{j n}\right) \\
& \quad+\left|\ell_{j+1, n} W U\right|(x)(W U)^{-1}\left(x_{j+1, n}\right) \leq C, \quad x \in \mathbf{I}_{j} .
\end{aligned}
$$

Proof. Notice that

$$
\ell_{j n}(x)=\frac{K_{n}\left(x, x_{j n}\right)}{K_{n}\left(x_{j n}, x_{j n}\right)},
$$

where $K_{n}(x, t)=\sum_{k=0}^{n} p_{k}(x) p_{k}(t)$ is the $n^{\text {th }}$ reproducing kernel function. Applying the Cauchy-Schwarz inequality to $K_{n}(x, t)$, we obtain

$$
\begin{aligned}
\left|\ell_{j n} W U\right|(x)(W U)^{-1}\left(x_{j n}\right) & \leq\left(\frac{K_{n}(x, x)(W U)^{2}(x)}{K_{n}\left(x_{j n}, x_{j n}\right)(W U)^{2}\left(x_{j n}\right)}\right)^{1 / 2} \\
& =\left(\frac{\lambda_{n}^{-1}\left((W U)^{2} ; x\right)(W U)^{2}(x)}{\lambda_{n}^{-1}\left((W U)^{2} ; x_{j n}\right)(W U)^{2}\left(x_{j n}\right)}\right)^{1 / 2} .
\end{aligned}
$$

By Lemma 6 and (28), we see $\mathbf{I}_{j} \subset \mathbf{J}_{L, n}^{*}, 1 \leq j \leq n$. Now applying the Christoffel function bounds of Corollary 1 (a) and (b), it follows from the above relation that

$$
\begin{aligned}
\left|\ell_{j n} W U\right|(x)(W U)^{-1}\left(x_{j n}\right) & \\
\leq & C\left(\frac{\varphi_{n}^{*}\left(x_{j n}\right)}{\varphi_{n}^{*}(x)}\right)^{1 / 2} \\
& \cdot \frac{\left(\bar{U}_{n}(x)\right)^{-2} U^{2}(x)}{\left(U_{n}^{*}\left(x_{j n}\right) W^{*}\left(x_{j n}\right)\right)^{-2}(W U)^{2}\left(x_{j n}\right)}, \quad x \in \mathbf{I}_{j} .
\end{aligned}
$$

According to the definition of $W^{*}$,

$$
W^{*}(x)=\left(1-x^{2}\right)^{p_{0}} W(x),
$$

and then

$$
U_{n}^{*}(x) W^{*}(x)=\left(1-x^{2}\right)^{p_{0}}\left[\left(1-x^{2}\right)^{1 / 2}+\frac{1}{n}\right]^{-2 p_{0}} \bar{U}_{n}(x) W(x),
$$

which by (2.23) in [7] for $x \in \mathbf{J}_{L, n}^{*}$ gives

$$
U_{n}^{*}(x) W^{*}(x) \sim \bar{U}_{n}(x) W(x) .
$$

It follows from (48) that for large enough $n$,

$$
\begin{aligned}
\left|\ell_{j n} W U\right|(x)(W U)^{-1}\left(x_{j n}\right) & \leq C\left(\frac{\varphi_{n}^{*}\left(x_{j n}\right)}{\varphi_{n}^{*}(x)}\right)^{1 / 2} \frac{\left(\bar{U}_{n}(x)\right)^{-2} U^{2}(x)}{\left(\bar{U}_{n}\left(x_{j n}\right)\right)^{-2} U^{2}\left(x_{j n}\right)} \\
& \leq C\left(\frac{\varphi_{n}^{*}\left(x_{j n}\right)}{\varphi_{n}^{*}(x)}\right)^{1 / 2}, \quad x \in \mathbf{I}_{j},
\end{aligned}
$$

as when $r=2$,

$$
\frac{\left(\bar{U}_{n}(x)\right)^{-2} U^{2}(x)}{\left(\bar{U}_{n}\left(x_{j n}\right)\right)^{-2} U^{2}\left(x_{j n}\right)} \sim 1 .
$$

Further, applying Theorem 5.7(b) in [1], we conclude for $x \in \mathbf{I}_{j}$,

$$
\varphi_{n}^{*}\left(x_{j n}\right) \sim \varphi_{n}^{*}(x)
$$

so that

$$
\left|\ell_{j n} W U\right|(x)(W U)^{-1}\left(x_{j n}\right) \leq C, \quad x \in \mathbf{I}_{j},
$$

and with a similar discussion, we also have

$$
\left|\ell_{j+1, n} W U\right|(x)(W U)^{-1}\left(x_{j+1, n}\right) \leq C, \quad x \in \mathbf{I}_{j} .
$$

This proves (42).

\section{Proof of Theorems}

3.1. Proof of Theorem 2. It is easy to check that $Q^{*}: \mathbf{I} \longrightarrow[0, \infty)$ is convex with $Q^{*}(a+)=Q^{*}(b-)=\infty$ and $Q^{*}(x)>Q^{*}(0)=0, x \in I \backslash\{0\}$, so by considering Lemma $3, W^{*}$ satisfies the assumptions about $W$. Furthermore, for $P \in \mathscr{P}_{t-\rho^{*}-(2 / p)}$, 


$$
P U^{*} \in \mathscr{P}_{t-2 / p}
$$

Observe that

$$
U(x) W(x)=U^{*}(x) W^{*}(x) .
$$

Then, applying Lemma 1, we obtain the results.

\subsection{Proof of Theorem 3}

(a) By Lemma 3, $W^{*} \in \mathscr{F}(\operatorname{Lip}(1 / 2))$. For $P \in \mathscr{P}_{t-\rho^{*}}$, we have $P U^{*} \in \mathscr{P}_{t}$. Thus, by (55), relation (23) follows from (32).

(b) If $W^{*} \in \mathscr{F}$ (Dini), then with the similar discussion as (a) and using (33), we prove that the statement of (b) is valid. So, it is necessary to prove that if $W \in \mathscr{F}$ (Dini), then $W^{*} \in \mathscr{F}$ (Dini).

The properties of $(a)-(e)$ in Definition 3 hold for $W^{*}$ if $W \in \mathscr{F}$ (Dini) because of the same argument as in the proof of Lemma 2.13 in [7] since properties of $(a)-(e)$ in Definition 3 are the same for both $\mathscr{F}(\operatorname{Lip}(1 / 2))$ and $\mathscr{F}(\mathrm{Dini})$. We will prove that the property of $(f)$ in Definition 3 also holds for $W^{*}$.

By (2.38) in [7], we have

$$
\begin{aligned}
S:= & \int_{x-\epsilon_{1}|x| / T^{*}(x)}^{x+\epsilon_{1}|x| / T^{*}(x)} \frac{Q^{* \prime}(s)-Q^{* \prime}(x)}{s-x} \mathrm{~d} s \\
\leq & \int_{x-\epsilon_{1}|x| /[(1-\mu) T(x)]}^{x+\epsilon_{1}|x| /[(1-\mu) T(x)]} \frac{Q^{* \prime}(s)-Q^{* \prime}(x)}{s-x} \mathrm{~d} s \\
= & \int_{x-\epsilon_{1}|x| /[(1-\mu) T(x)]}^{x+\epsilon_{1}|x| /[(1-\mu) T(x)]} \frac{Q^{* \prime}(s)-Q^{* \prime}(x)}{s-x} \mathrm{~d} s \\
& +p_{0} \int_{x-\epsilon_{1}|x| /[(1-\mu) T(x)]}^{x+\epsilon_{1}|x| /[(1-\mu) T(x)]} \frac{q^{\prime}(s)-q^{\prime}(x)}{s-x} \mathrm{~d} s \\
= & S_{1}+S_{2} .
\end{aligned}
$$

According to Definition $3(f)$,

$$
S_{1} \leq C\left|Q^{\prime}(x)\right| \text {. }
$$

Meanwhile, using Corollary 2.1 (a) in [7], for $s \in[x-$ $\left.\epsilon_{1}|x| /[(1-\mu) T(x)] x+\epsilon_{1}|x| /[(1-\mu) T(x)]\right]$, we see

$$
\begin{aligned}
\frac{1}{1-s^{2}}-\frac{1}{1-x^{2}} & =\frac{s^{2}-x^{2}}{\left(1-x^{2}\right)\left(1-s^{2}\right)} \leq \frac{2|s-x|}{\left(1-x^{2}\right)\left(1-s^{2}\right)} \\
& \leq \frac{2 \epsilon_{1}|x|}{(1-\mu) T(x)\left(1-x^{2}\right)\left(1-s^{2}\right)} \\
& \leq \frac{2 \epsilon_{1}}{\epsilon(1-\mu)\left(1-s^{2}\right)}=\frac{1}{2\left(1-s^{2}\right)},
\end{aligned}
$$

where $\epsilon_{1}=\epsilon(1-\mu) / 4$ and $\epsilon$ and $\mu$ are shown in (2.29) and (1.23) in [7], respectively, and hence

$$
\frac{1}{1-s^{2}} \leq \frac{2}{1-x^{2}} .
$$

Using this relation and

$$
q^{\prime}(x)=\frac{2 x}{1-x^{2}}
$$

we obtain

$$
\begin{aligned}
S_{2} & =p_{0} \int_{x-\epsilon_{1}|x| /[(1-\mu) T(x)]}^{x+\epsilon_{1}|x| /[(1-\mu) T(x)]} \frac{2(1+x s)}{\left(1-x^{2}\right)\left(1-s^{2}\right)} \mathrm{d} s \\
& \leq \frac{8\left|p_{0}\right|}{\left(1-x^{2}\right)^{2}} \int_{x-\epsilon_{1}|x| /[(1-\mu) T(x)]}^{x+\epsilon_{1}|x| /[(1-\mu) T(x)]} \mathrm{d} s \\
& \leq \frac{C|x|}{\left(1-x^{2}\right)} \cdot \frac{1}{\left(1-x^{2}\right) T(x)} .
\end{aligned}
$$

By (2.30) and (2.35) in [7], we further get

$$
S_{2} \leq C\left|q^{\prime}(x)\right| \leq C\left|Q^{\prime}(x)\right| \text {. }
$$

Substituting $S_{1}$ and $S_{2}$ into $S$ gives

$$
S \leq C\left|Q^{\prime}(x)\right| .
$$

Thus, by (2.35) in [7], we infer that

$$
S \leq C\left|Q^{* \prime}(x)\right| \text {. }
$$

This proves property $(f)$ of Definition 3 .

3.3. Proof of Theorem 4. (a) The proof is similar to Theorem 1.7 in [6], but we provide the details with modification. Denote by $\left\{\ell_{k n}\right\}_{k=1}^{n}$ the fundamental polynomials of Lagrange interpolation at the zeros $\left\{x_{k n}\right\}_{k=1}^{n}$ of the orthogonal polynomials $p_{n}\left((W U)^{2}, x\right)$ for the weight $(W U)^{2}$.

Recall (5); the infimum is actually attained when we take $P$ to be $\ell_{k n} \in \mathbf{P}_{n-1}$ satisfying $\ell_{k n}\left(x_{j n}\right)=\delta_{k j}$. So, a classical Gauss quadrature formula for the weight $(W U)^{2}$ is

$$
\lambda_{n}\left((W U)^{2} ; x_{k n}\right)=\int_{\mathrm{I}} \ell_{k n}^{2}(W U)^{2} .
$$

By Lemma 11.8 in [1], (pp. 320-321) and relation (55), we infer that

$$
\begin{aligned}
\lambda_{n}\left((W U)^{2} ; x_{k n}\right) W^{*}\left(x_{k n}\right)^{-2}+\lambda_{n}\left((W U)^{2} ; x_{k+1, n}\right) W^{*}\left(x_{k+1, n}\right)^{-2} \\
=\int_{I}\left[\ell_{k n}(t)^{2} W^{*}\left(x_{k n}\right)^{-2}+\ell_{k+1, n}(t)^{2} W^{*}\left(x_{k+1, n}\right)^{-2}\right] \\
\quad \cdot W^{*}(t)^{2} U^{*}(t)^{2} \mathrm{~d} t \\
\geq \int_{x_{k+1, n}}^{x_{k n}}\left[\ell_{k n}(t)^{2} W^{*}\left(x_{k n}\right)^{-2}+\ell_{k+1, n}(t)^{2} W^{*}\left(x_{k+1, n}\right)^{-2}\right] \\
\quad \cdot W^{*}(t)^{2} U^{*}(t)^{2} \mathrm{~d} t \\
\geq \frac{1}{2} \int_{x_{k+1, n}}^{x_{k n}} U^{*}(t)^{2} \mathrm{~d} t .
\end{aligned}
$$


On the other hand, according to Lemma 6 and Theorem $5, \mathbf{I}_{k} \subset \mathbf{J}_{L, n}^{*}$, so that by (20),

$$
\begin{aligned}
& \lambda_{n}\left((W U)^{2} ; x_{k n}\right) W^{*}\left(x_{k n}\right)^{-2}+\lambda_{n}\left((W U)^{2} ; x_{k+1, n}\right) W^{*}\left(x_{k+1, n}\right)^{-2} \\
& \quad \leq c\left[\varphi_{n}^{*}\left(x_{k n}\right) U_{n}^{*}\left(x_{k n}\right)^{2}+\varphi_{n}^{*}\left(x_{k+1, n}\right) U_{n}^{*}\left(x_{k+1, n}\right)^{2}\right] .
\end{aligned}
$$

Let $j=j(k)$ be $j$ defined by (34); then by (51) and Lemma 4, we get

$$
\begin{aligned}
& c \varphi_{n}^{*}\left(x_{k n}\right)\left[U_{n}^{*}\left(x_{k n}\right)^{2}+U_{n}^{*}\left(x_{k+1, n}\right)^{2}\right] \\
& \geq \int_{x_{k+1, n}}^{x_{k n}} U^{*}(t)^{2} \mathrm{~d} t \sim \int_{x_{k+1, n}}^{x_{k n}}\left|t-t_{j}\right|^{2 p_{j}^{*}} \mathrm{~d} t \prod_{i=1, i \neq j}^{r}\left|x_{k n}-t_{i}\right|^{2 p_{i}^{*}},
\end{aligned}
$$

where $p_{j}^{*}=p_{j(k)}^{*}, p_{i}^{*}=p_{i}$, if $2 \leq i \leq r-1, p_{1}^{*}=p_{1}-p_{0}$, and $p_{r}^{*}=p_{r}-p_{0}$. Also, we have

$$
\begin{aligned}
U_{n}^{*}\left(x_{k n}\right)^{2}+U_{n}^{*}\left(x_{k+1, n}\right)^{2} & \sim\left[\left(\left|x_{k n}-t_{j}\right|+\frac{1}{n}\right)^{2 p_{j}^{*}}+\left(\left|x_{k+1, n}-t_{j}\right|+\frac{1}{n}\right)^{2 p_{j}^{*}}\right] \\
& \cdot \prod_{i=1, i \neq j}^{r}\left(\left|x_{k n}-t_{i}\right|+\frac{1}{n}\right)^{2 p_{i}^{*}},
\end{aligned}
$$

and by (35), we further get

$$
\begin{aligned}
U_{n}^{*}\left(x_{k n}\right)^{2}+U_{n}^{*}\left(x_{k+1, n}\right)^{2} & \sim\left[\left(\left|x_{k n}-t_{j}\right|+\frac{1}{n}\right)^{2 p_{j}^{*}}+\left(\left|x_{k+1, n}-t_{j}\right|+\frac{1}{n}\right)^{2 p_{j}^{*}}\right] \\
& \cdot \prod_{i=1, i \neq j}^{r}\left|x_{k n}-t_{i}\right|^{2 p_{i}^{*}} .
\end{aligned}
$$

By (68) and (70), we get the following relation after simplifying by $\prod_{i=1, i \neq j}^{r}\left|x_{k n}-t_{i}\right|^{2 p_{i}^{*}}$ :

$$
\begin{aligned}
& \int_{x_{k+1, n}}^{x_{k n}}\left|t-t_{j}\right|^{2 p_{j}^{*}} \mathrm{~d} t \\
& \quad \leq c \varphi_{n}^{*}\left(x_{k n}\right)\left[\left(\left|x_{k n}-t_{j}\right|+\frac{1}{n}\right)^{2 p_{j}^{*}}+\left(\left|x_{k+1, n}-t_{j}\right|+\frac{1}{n}\right)^{2 p_{j}^{*}}\right] .
\end{aligned}
$$

In fact, for $x, y \in \mathbf{I}_{k}$, using (2.8) in [6] and following the argument in the proof of Lemma 5 in [6], we can obtain (1$x)^{p_{1}-p_{0}} \sim(1-x+(1 / n))^{p_{1}-p_{0}} \sim(1-y)^{p_{1}-p_{0}} \sim(1-y+(1 /$ $n))^{p_{1}-p_{0}}$ and $(1+x)^{p_{r}-p_{0}} \sim(1+x+(1 / n))^{p_{r}-p_{0}} \sim(1+y)^{p_{r}-p_{0}}$ $\sim(1+y+(1 / n))^{p_{r}-p_{0}}$, so $(71)$ can be written as

$$
\begin{aligned}
& \int_{x_{k+1, n}}^{x_{k n}}\left|t-t_{j}\right|^{2 p_{j}} \mathrm{~d} t \\
& \quad \leq c \varphi_{n}^{*}\left(x_{k n}\right)\left[\left(\left|x_{k n}-t_{j}\right|+\frac{1}{n}\right)^{2 p_{j}}+\left(\left|x_{k+1, n}-t_{j}\right|+\frac{1}{n}\right)^{2 p_{j}}\right],
\end{aligned}
$$

where $2 \leq j \leq r-1$.

Further, by (36),

$$
\begin{aligned}
\int_{x_{k+1, n}}^{x_{k n}}\left|t-t_{j}\right|^{2 p_{j}} \mathrm{~d} t \leq & c \varphi_{n}^{*}\left(x_{k n}\right)\left\{\left[\left|x_{k n}-t_{j}\right|+\varphi_{n}^{*}\left(x_{k n}\right)\right]^{2 p_{j}}\right. \\
+ & {\left.\left[\left|x_{k+1, n}-t_{j}\right|+\varphi_{n}^{*}\left(x_{k n}\right)\right]^{2 p_{j}}\right\} . }
\end{aligned}
$$

By calculation from (73), we get

$$
\begin{gathered}
\frac{1}{2 p_{j}+1}|| x_{k n}-\left.t_{j}\right|^{2 p_{j}+1}+\sigma\left|x_{k+1, n}-t_{j}\right|^{2 p_{j}+1} \mid \\
=\int_{x_{k+1, n}}^{x_{k n}}\left|t-t_{j}\right|^{2 p_{j}} \mathrm{~d} t \\
\leq c \varphi_{n}^{*}\left(x_{k n}\right)\left\{\left[\left|x_{k n}-t_{j}\right|+\varphi_{n}^{*}\left(x_{k n}\right)\right]^{2 p_{j}}\right. \\
\left.+\left[\left|x_{k+1, n}-t_{j}\right|+\varphi_{n}^{*}\left(x_{k n}\right)\right]^{2 p_{j}}\right\},
\end{gathered}
$$

where

$$
\sigma= \begin{cases}1, & t_{j} \in \mathbf{I}_{k}, \\ -1, & t_{j} \notin \mathbf{I}_{k} .\end{cases}
$$

We distinguish two cases.

Case 1. $p_{j} \geq 0$. By Lemma 2.6 in [6], we assert that if $p \geq 0, B_{n} \geq A_{n} \geq 0, C_{n} \geq 0, \sigma= \pm 1$, and $B_{n}^{p+1}+\sigma A_{n}^{p+1} \leq$ $C C_{n}\left[\left(B_{n}+C_{n}\right)^{p}+\left(A_{n}+C_{n}\right)^{p}\right]$, then $B_{n}+\sigma A_{n} \leq c C_{n}$.

Using this inequality, it follows from (74) that

$$
x_{k n}-x_{k+1, n} \leq c \varphi_{n}^{*}\left(x_{k n}\right) .
$$

Case 2. $-1 / 2<p_{j}<0$. By (74),

$$
\begin{aligned}
& \frac{1}{2 p_{j}+1}|| x_{k n}-\left.t_{j}\right|^{2 p_{j}+1}+\sigma\left|x_{k+1, n}-t_{j}\right|^{2 p_{j}+1} \mid \\
& =\int_{x_{k+1, n}}^{x_{k n}}\left|t-t_{j}\right|^{2 p_{j}} \mathrm{~d} t \\
& \leq c_{0} \varphi_{n}^{*}\left(x_{k n}\right) \min \left\{\varphi_{n}^{*}\left(x_{k n}\right)^{2 p_{j}},\left|x_{k+1, n}-t_{j}\right|^{2 p_{j}},\left|x_{k, n}-t_{j}\right|^{2 p_{j}}\right\} .
\end{aligned}
$$

Case 2.1. $t_{j} \in \mathbf{I}_{k}$. Inequality (77) gives

$$
\left|x_{\kappa n}-t_{j}\right|^{2 p_{j}+1} \leq c \varphi_{n}^{*}\left(x_{k n}\right)^{2 p_{j}+1}, \quad \kappa=k, k+1,
$$

which yields (76).

Case 2.2. $t_{j} \notin \mathbf{I}_{k}$. In this case, we distinguish two subcases. Suppose without loss of generality that $x_{k+1, n}>t_{j}$.

Case 2.2.1. If $\left|x_{k+1, n}-t_{j}\right| \geq 2 c_{0} \varphi_{n}^{*}\left(x_{k n}\right)$, where $c_{0}$ is given by (77), then 


$$
\begin{aligned}
\int_{x_{k+1, n}}^{x_{k n}}\left(t-t_{j}\right)^{2 p_{j}} \mathrm{~d} t & =\int_{x_{k+1, n}}^{x_{k n}}\left(t-t_{j}\right)\left(t-t_{j}\right)^{2 p_{j}-1} \mathrm{~d} t \\
& \geq\left(x_{k+1, n}-t_{j}\right) \int_{x_{k+1, n}}^{x_{k n}}\left(t-t_{j}\right)^{2 p_{j}-1} \mathrm{~d} t=\left(x_{k+1, n}-t_{j}\right) \frac{1}{2\left|p_{j}\right|}\left[\left(x_{k+1, n}-t_{j}\right)^{2 p_{j}}-\left(x_{k n}-t_{j}\right)^{2 p_{j}}\right] \\
& \geq \frac{c_{0} \varphi_{n}^{*}\left(x_{k n}\right)}{\left|p_{j}\right|}\left[\left(x_{k+1, n}-t_{j}\right)^{2 p_{j}}-\left(x_{k n}-t_{j}\right)^{2 p_{j}}\right]
\end{aligned}
$$

which by (77) gives

$$
\left(x_{k+1, n}-t_{j}\right)^{2 p_{j}} \leq\left(1-\left|p_{j}\right|\right)^{-1}\left(x_{k n}-t_{j}\right)^{2 p_{j}} \leq 2\left(x_{k n}-t_{j}\right)^{2 p_{j}} \text {. }
$$

On the other hand, by (77)-(80),

$$
\begin{aligned}
& c_{0} \varphi_{n}^{*}\left(x_{k n}\right)\left(x_{k+1, n}-t_{j}\right)^{2 p_{j}} \\
& \quad \geq \int_{x_{k+1, n}}^{x_{k n}}\left(t-t_{j}\right)^{2 p_{j}} \mathrm{~d} t \geq\left(x_{k n}-t_{j}\right)^{2 p_{j}}\left(x_{k n}-x_{k+1, n}\right) \\
& \quad \geq \frac{1}{2}\left(x_{k+1, n}-t_{j}\right)^{2 p_{j}}\left(x_{k n}-x_{k+1, n}\right),
\end{aligned}
$$

and hence (76) follows.

Case 2.2.2. $\left|x_{k+1, n}-t_{j}\right|<2 c_{0} \varphi_{n}^{*}\left(x_{k n}\right)$. By (77),

$$
\begin{aligned}
& c_{0} \varphi_{n}^{*}\left(x_{k n}\right)^{2 p_{j}+1} \\
& \geq \frac{1}{2 p_{j}+1}\left[\left(x_{k n}-t_{j}\right)^{2 p_{j}+1}-\left(x_{k+1, n}-t_{j}\right)^{2 p_{j}+1}\right] \\
& \geq \frac{1}{2 p_{j}+1}\left[\left(x_{k n}-t_{j}\right)^{2 p_{j}+1}-\left(2 c_{0} \varphi_{n}^{*}\left(x_{k n}\right)\right)^{2 p_{j}+1}\right] .
\end{aligned}
$$

So, $x_{k n}-t_{j} \leq c \varphi_{n}^{*}\left(x_{k n}\right)$ and (76) follows.

(b) Now, let us prove (27). We must prove that for some constant $c>0$ and $n$ large enough, we have

$$
x_{k n}-x_{k+1, n} \geq c \varphi_{n}^{*}\left(x_{k n}\right), \quad k=1,2, \ldots, n-1 .
$$

First, by our Markov-Bernstein inequality (23) and Lemma 7, we have that

$$
\begin{aligned}
& \left\|\left(\ell_{k n} W U\right)^{\prime} \varphi_{n}^{*}\right\|_{L_{\infty}(\mathrm{I})}(W U)^{-1}\left(x_{k n}\right) \\
& \quad \leq C_{1}\left\|\ell_{k n} W U\right\|_{L_{\infty}(\mathrm{I})}(W U)^{-1}\left(x_{k n}\right) \leq C .
\end{aligned}
$$

Then, by the mean value theorem, for some $\xi$ between $x_{k n}$ and $x_{k+1, n}$,

$$
\begin{aligned}
1= & \left(\ell_{k n} W U\right)\left(x_{k n}\right)(W U)^{-1}\left(x_{k n}\right) \\
& -\left(\ell_{k n} W U\right)\left(x_{k+1, n}\right)(W U)^{-1}\left(x_{k n}\right) \\
= & \left(\ell_{k n} W U\right)^{\prime}(\xi)(W U)^{-1}\left(x_{k n}\right)\left(x_{k n}-x_{k+1, n}\right) \\
\leq & C\left(\varphi_{n}^{*}\right)^{-1}(\xi)\left(x_{k n}-x_{k+1, n}\right) .
\end{aligned}
$$

Thus, by (51), we get the lower bound and finish the proof of (b).

3.4. Proof of Theorem 6. By Lemma 3, $W^{*} \in \mathscr{F}(\operatorname{Lip}(1 / 2))$. Then, following the argument in the proof of Theorem 5 , the statements of Theorem 6 follow directly from Theorem 1.10 in [6].

\section{Data Availability}

No data were used to support this study.

\section{Conflicts of Interest}

The author declares that there are no conflicts of interest.

\section{Acknowledgments}

This research was supported in part by the National Natural Science Foundation of China (no. 11626060) and Scientific Research Fund of Fujian Provincial Education Department (no. JAT160172).

\section{References}

[1] A. L. Levin and D. S. Lubinsky, Orthogonal Polynomials for Exponential Weights, Springer, New York, NY, USA, 2001.

[2] A. L. Levin and D. S. Lubinsky, "Christoffel functions and orthogonal polynomials for exponential weights on $[-1,1]$," Memoirs of the American Mathematical Society, vol. 11, no. $535,1994$.

[3] A. L. Levin and D. S. Lubinsky, "Orthogonal polynomials for exponential weights $x^{2 \rho} e^{-2 Q(x)}$ on $[0, d)$," Approximation Theory, vol. 134, pp. 199-256, 2005.

[4] A. L. Levin and D. S. Lubinsky, "Orthogonal polynomials for exponential weights $x^{2 \rho} e^{-2 Q(x)}$ on $\mathrm{I}[0, d)$," Approximation Theory, vol. 139, pp. 107-143, 2006.

[5] T. Kasuga and R. Sakai, "Orthonormal polynomials with generalized Freud-type weights," Journal of Approximation Theory, vol. 121, no. 1, pp. 13-53, 2003.

[6] R. Liu and Y. G. Shi, "The zeros of orthogonal polynomials for Jacobi-exponential weights, abstract and applied analysis," Article ID 386359, 17 pages, 2012.

[7] R. Liu and Y. G. Shi, "Generalized christoffel functions for Jacobi-exponential weights on $[-1,1]$," Acta Mathematica Hungarica, vol. 148, no. 1, pp. 17-42, 2016.

[8] Y. G. Shi, "Generalized Christoffel functions for Jacobi-exponential weights," Acta Mathematica Hungarica, vol. 140, no. 1-2, pp. 71-89, 2013. 
[9] Y. G. Shi, "Orthogonal polynomials for Jacobi-exponential weights $\left(1-x^{2}\right)^{\rho} e^{-Q(x)}$ on $(-1,1), "(-1,1)$ Acta Mathematica Hungarica, vol. 140, no. 4, pp. 363-376, 2013.

[10] G. Mastroianni and V. Totik, "Uniform spacing of zeros of orthogonal polynomials," Constructive Approximation, vol. 32, no. 2, pp. 181-192, 2010.

[11] G. Mastroianni and I. Notarangelo, "Lagrange interpolation with exponential weights on $(-1,1)$," Approximation Theory, vol. 167, pp. 65-93, 2013.

[12] G. Mastroianni and I. Notarangelo, "Lagrange interpolation at Pollaczek-Laguerre zeros on the real semiaxis," Journal of Approximation Theory, vol. 245, pp. 83-100, 2019. 